\title{
Editorial
}

\section{In need of publishing a paper to find a job or grant? Co-authorship without contributing: The rise of an ethical problem}

In recent times the pressure for publishing has led researchers to wanting to appear in the author list without having made a significant conntribution, a practice not included in the Vancouver Agreement that frames who the authors of a paper should be. This situation creates not only an ethical problem but also a problem within the work environment if a researcher refuses to include authors who have not participated in the study, creating unconfortable situations in some institutions.

It is well known that some researchers that appear to be very productive, holding a high record of plublished papers, may have entered the ratings without having read a manuscript that later mentions them in the authorship list, thanks to supporting infrastructure or by maintaining networks. This network somehow strengthens the group leader, or leader of the line and also some "emerging researchers" so they can apply for more grants and increase their chances of finding better jobs or more grants for their students. Some professors even take advantage of their students' novel ideas to generate a grant and thus maintain the vanguard, without even including the latter in the benefits of the grant or the paper that is generated. Neither European, North American nor Chilean universities are exempt from these practices. In Chile, institutions such as Universidad de Chile, Pontificia Universidad Católica de Chile, and Universidad Austral de Chile have had a series of problems with postgraduate students denouncing their professors for using their theses, or part of these,to generate papers without including them in the authorship list. This unethical practice has some deep roots, fueled by the desire of some proffesors to remain in the "elite" of research.

The solution to this problem is complex since grants are scarce and competition is tough. In some research centers and universities this subject is not even spoken of, because this practice is highly institutionalised, therefore discusing the ethics of this matter or the Vancouver Agreement is not an option. The only way to eradicate these practices would be to generate debate and denounce them, and to avoid "collaborating" with this type of researchers.

Unfortunately, science is not estranged from what happens in the world, nor within non-academic institutions, where this type of action is common and very recurrent. Science should be a beacon for scociety, a place to look toward and be guided by.

Editorial Committee Austral Journal of Veterinary Sciences 